\title{
Research on TSP Solution Based on Improved Simulated Annealing Algorithm
}

\author{
Anzhi Qi ${ }^{1, a}$ \\ ${ }^{1}$ Liaoning Jianzhu Vocational College, Liaoyang, 111000, China \\ ${ }^{\mathrm{a} e m a i l}$
}

Keywords: TSP Solution, Simulated Annealing Algorithm, Acceptable rule

\begin{abstract}
Travelling Salesman Problem (TSP) is a typical combinatorial optimization problem. The main idea of the problem is that given a number of cities and the distances between each city and tries to get the shortest path that visits each city exactly once and back to the starting point. This report introduces multiple arithmetic operators to improve the simulated annealing algorithm to apply in the solution of TSP. The result shows that the improved simulated annealing algorithm is effective and efficient.
\end{abstract}

\section{Introduction}

TSP, the traveling salesman problem, is one of the most famous problems in the field of mathematics. Suppose there is a traveling merchant who wants to visit $\mathrm{N}$ cities, and he must choose the path he wants to go. The limitation of the path is that every city can only visit once, and finally go back to the original city. The path selection objective is the required path distance, which is the minimum value among all paths. The traveling salesman problem (TSP), also known as the traveling salesman problem, mathematical problem proposed by Sir William Rowan Hamilton and the Irish mathematician Penyngton British mathematician Thomas Kirkman in nineteenth Century, it refers to a $\mathrm{n}$ between a given city and give each of the two city distance traveling salesman must visit to all the shortest path the city once and only once, and return to the original starting point, it has been demonstrated that it belongs to the NP problem. The first formal algorithm to solve the TSP problem in history was born in 1954, and it was used to calculate the TSP problem in 49 cities. Up to now, the most advanced computer technology has been used to solve the TSP problem of 24978 cities. Because the problem is simply described, and the actual model in the drilling line scheme, the printed circuit board of chain stores, goods distribution network routing optimization problems and has been widely applied, it has long been attracting many researchers at home and abroad to study, they try to use all kinds of algorithms to solve TSP problems. Summed up: approximate solution, local search method, neural network, genetic algorithm, clonal algorithm, simulated annealing algorithm and hybrid genetic algorithms.

\section{Simulated Annealing Algorithm and Its Improvement}

Simulated Annealing. The simulated annealing algorithm (Simulated Annealing) was first seen in IBM Thomas.J. Watson research center in the article by S.Kirkpatrick et al, they studied the optimization of the portfolio, according to the iterative improvement proposed simulated annealing algorithm, simulated annealing algorithm has strong local search ability. The simulated annealing algorithm is derived from the principle of solid annealing, which heats the solid to a sufficiently high level, and then cools it down slowly to achieve the lowest energy point. Conversely, if the rapid cooling, it cannot reach the lowest point. During heating, temperature rise inside the solid particles with the shape into disorder, can be increased, and the slow cooling when the particle is orderly, temperature has reached equilibrium in each state, and finally reached the ground state at room temperature can be reduced to a minimum. According to the Metropolis criterion, the probability that the particle tends to equilibrium at the temperature T is exp (-E/ (kT)), where E is the internal energy at the temperature $\mathrm{T}, \mathrm{E}$ is the change, and $\mathrm{K}$ is the Boltzman constant. 
With the solid simulated annealing combinatorial optimization problems, to be able to E simulation as the objective function value of $\mathrm{F}$, temperature of $\mathrm{T}$ evolved into $\mathrm{t}$ control parameters, to obtain the solution of simulated annealing algorithm in combinatorial optimization problems: the initial solution I and initial $\mathrm{t}$ control parameters, repeat a target function iterative calculation, difference to accept or reject" in the current solution, and gradually decrease the value of $\mathrm{T}$, the algorithm terminates when the current solution is the approximate optimal solution, which is a heuristic random search method based on Monte Carlo iterative process. The annealing process is controlled by the cooling schedule (Cooling Schedule), including the initial value of the control parameter $\mathrm{T}$ and its attenuation factor $\mathrm{A}$, the number of iterations $\mathrm{L}$ and the stopping condition $\mathrm{C}$ at each $t$ value. We can write the simulated annealing algorithm to solve the TSP problem through the above idea. Steps are as follows:

(1): initialize the initial temperature of $\mathrm{T}$ (sufficiently large), the initial solution s (randomly selected a TSP route, the route length calculated through Cost (s) as the evaluation function, this is the starting point of the iterative method), the number of iterations in $\mathrm{L}$ for each $\mathrm{T}$ value;

(2) do $k=1$ (3) to sixth steps to $k=L$;

(3) generate a news' (usually using the 2-opt algorithm to generate new paths);

(4) calculate increment Cost=Cost (s') -Cost (s), where Cost (s) is the evaluation function;

(5) if $t '<0, s$ ' is accepted as the new current solution, otherwise the probability exp $(-t$ ' $/ T)$ is accepted as the new current solution of s';

(6) if the termination condition is satisfied, the current solution is output as the optimal solution and the end program is finished. Termination conditions are usually taken when successive new solutions are not accepted;

(7) T gradually decreases, and $\mathrm{T}$ tends to 0 , and then turns to second steps.

Improvement of Simulated Annealing. The simulated annealing algorithm selects an optimal solution from the nearest solution space of the current solution as the current solution until a local optimal solution is reached. Hill climbing algorithm is very simple, the main drawback is that it will fall into the local optimal solution, and not necessarily can find the global optimal solution. If the $\mathrm{C}$ point is the current solution, the hill climbing algorithm searches for the A point, and the local optimal solution will stop searching, because at the A point no matter how small the direction is, no better solution can be obtained. The hill-climbing method is completely greedy method, every time a short-sighted choice of the current optimal solution, therefore only searching local optimal value. The improvement of the improved simulated annealing algorithm is to introduce a variety of operators (such as: shift, exchange, inversion, etc.) to generate new solutions. And with certain probability to decide which operator to use to generate new solution space. In the operator, the main idea of inversion is: randomly select two cities in the path, and then the city order between the two cities is completely inverted to get a new path. As the example above: two different number of $\mathrm{K}$ and $\mathrm{m}$ (assuming $\mathrm{k}<\mathrm{m}$ ), is the original path ( $\mathrm{w} 1, \mathrm{w} 2, \ldots \mathrm{wk}-1$, wk, wk $+1, \ldots \mathrm{wm}-1, \mathrm{wm}, \mathrm{wm}+1, \ldots \mathrm{wn}$ ) becomes a new path: (w1, w2, .. wk- 1 , wm, wm- $1, \ldots w k+1, w k, w m+1, \ldots w n)$. the main idea of the shift is to randomly select two cities and move the cities between the two cities to one right. for example, the city wk and wm (assuming $\mathrm{k}<\mathrm{m}$ ) are selected randomly, and the original path (w1, w2) is used, (wk-1, wk, wk+1, wm-1, wm, wm $+1, w m+2, \ldots w n)$ becomes a new path: (w1, w2, ... wk-1, $w m+1, w k, w k+1 \ldots w m-1, w m, w m+2, \ldots w n)$. the idea of exchange is the same as that of the 2-OPT algorithm, which generates two cities randomly and exchanges the two cities to produce new solutions. Simulated annealing is also a greedy algorithm, but its search process introduces random factors. The simulated annealing algorithm accepts a solution which is inferior to the current solution with a certain probability, so it is possible to jump out of the local optimal solution and achieve the global optimal solution.

\section{Applications of Improved Simulated Annealing Algorithm in TSP Solution}

Algorithm Process. Definite main function. The key parameters of the main function include initial temperature $\mathrm{T}=1000$, end temperature Tend $=0.001$, cooling rate $\mathrm{q}=0.9$ and chain length $\mathrm{L}=500$. 
And the function of each module is called, and the result is displayed on the MATLAB interface. Computing distance matrix. First of all, we need to calculate the distance between 22 cities given by $\mathrm{N}$, and use the distance formula in Mathematics: $\mathrm{D}(\mathrm{I}, \mathrm{J})=$. Initial solution. The initial solution of the algorithm is obtained by using Matlab's own function randperm. The initial solution of $\mathrm{N}$ cities is $\mathrm{S} 1=$ randperm (S1). Generating new solution. In this paper, we use 2-opt method to generate new solutions, and use the random numbers generated by the function a=round (rand $(1,2)(\mathrm{N}-1)+1)$ to exchange the order of the corresponding cities, and obtain new routes. Comparison of Metropolis criteria. A new solution is used to subtract the current solution, and if the result is less than zero, it is accepted; otherwise, the probability $\exp (-\mathrm{dC} / \mathrm{T})$ is used to judge whether to accept the new solution. Draw the path map, output path function, feasible solution path length function. The main function is run on MATLAB, and the initial solution, the optimal solution, the optimization process and the path are obtained.

Create City Coordinate Position. This paper first solves the N=14 problem of TSP, and obtains the initial solution, the optimal solution and the time required for the 14 cities by the following functions. Here are the coordinates of the 14 cities imported from the CityPosition1.mat file, as shown in Table 1.

Table 1. City coordinate positions

\begin{tabular}{|c|c|c|}
\hline & $\mathrm{x}$ & $\mathrm{y}$ \\
\hline 1 & 16.4700 & 96.1000 \\
\hline 2 & 16.4700 & 94.4400 \\
\hline 3 & 20.0900 & 92.5400 \\
\hline 4 & 22.3900 & 93.3700 \\
\hline 5 & 35.2300 & 97.2400 \\
\hline 6 & 22.0000 & 96.0500 \\
\hline 7 & 20.4700 & 97.0200 \\
\hline 8 & 17.2000 & 96.2900 \\
\hline 9 & 16.3000 & 97.3800 \\
\hline 10 & 14.0500 & 98.1200 \\
\hline 11 & 16.5300 & 97.3800 \\
\hline 12 & 21.5200 & 95.5900 \\
\hline 13 & 19.4100 & 97.1300 \\
\hline 14 & 20.0900 & 92.5500 \\
\hline
\end{tabular}

The distance between $\mathrm{N}$ cities is calculated by using the following program. The distance matrix is $(\mathrm{N} * \mathrm{~N})$, and the distance matrix is calculated as function $\mathrm{D}=$ Distanse $(\mathrm{a})$. Through the $\mathrm{a}=$ round (rand $(1,2) *(\mathrm{~N}-1)+1)$ statements in the following program randomly generate two positions, exchange the order between them, the new path is a new solution. For example, any number of two generated numbers is $\mathrm{x}, \mathrm{y}$, exchange $\mathrm{x}, \mathrm{y}$ order, then the corresponding $\mathrm{X}$ and $\mathrm{Y}$ location of the city exchange, get a new path. If the previous solution is $\mathrm{Ci}=(\mathrm{C} 1, \mathrm{C} 2 \ldots \mathrm{Cx}, \ldots \mathrm{Cy}, \ldots \mathrm{Cn})$, and the new path after exchange is $\mathrm{Ci}=(\mathrm{C} 1, \mathrm{C} 2, \ldots \mathrm{Cy}, \ldots \mathrm{Cx}, \ldots, \mathrm{Cn})$ is a new path, a new feasible solution $\mathrm{S} 2$. The Metropolis criterion is the key part of simulated annealing algorithm, which determines whether the algorithm converges to the global optimal solution or falls into the local minimum solution. The solutions generated by the Metropolis criterion cannot be optimal solutions, but the deterioration solutions are not uniformly rejected. The researchers adopt a set of function probability accept them to avoid the local optimal solution instead of the optimal solution that should converge to the global. Before the program gets the initial solutions and solutions, now algorithm requires trajectory function using route will finally get the initial solution and the optimal solution for image drawing, process optimization algorithm to solve the TSP problem has also appeared through the program, so that readers see the initial solution and the optimized solutions are different at a glance.

Operation Interface Diagram. This is a TSP optimization problem composed of 14 cities. This paper sets cooling rate $\mathrm{q}=0.9$, initial temperature $\mathrm{T} 0=1000$, end temperature Tend $=0.001$, chain length $\mathrm{L}=500$. In many practical operations, the solutions obtained are in line with the requirements 
of the problem, and the time is about 3.313s. In the previous 40 iterations, the change of the numerical value is not obvious, which may be the local optimal solution. However, as the number of iterations increases, the algorithm succeeds in searching the optimal solution in the global situation. The Metropolis criterion succeeds in accepting the solutions with a certain function probability, and accepts them as a new solution, avoiding the local optimal solution replacing the optimal solution that should converge to the global. The important value of Metropolis criterion in the algorithm is difficult to replace.

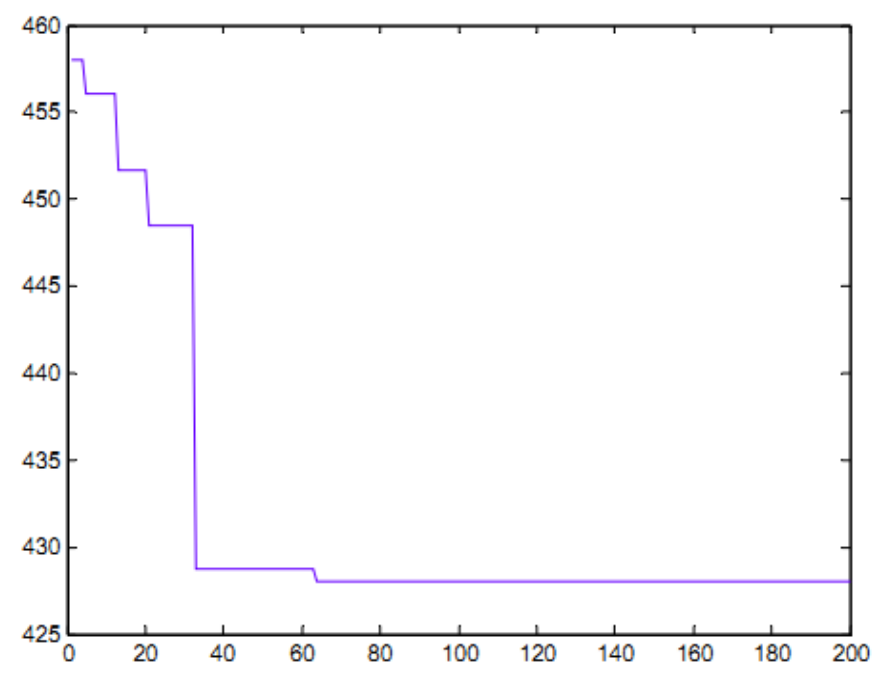

Figure 1. Optimization process figure

\section{Conclusion}

In this paper, the improved simulated annealing algorithm is used to run the optimal route problem of 14 cities and 34 cities, and the approximate solution is also in line with the requirements. At the same time, the operation time is also very short, which conforms to the actual application of efficient and accurate requirements. Meanwhile, simulated annealing algorithm is not omnipotent. People can improve algorithms according to their requirements. At the same time, those parameters are not immutable, need to change according to the actual situation. This requires artificial testing and debugging to the appropriate value to achieve the best solution requirements.

\section{References}

[1] Xu Xiaoping, Zhu Qiuqiu. Improved Simulated Annealing Algorithm of Solving TSP [J]. Computer Systems \& Applications, 2015, 24(12): 152-156.

[2] Liu Shiqing, Yang Kongyu. Improvement of genetic algorithm of TSP [J]. Journal of Beijing Information Science and Technology University, 2014, 29(2): 46-50.

[3] Zeng Xuhong, Zeng Guosun. Pricing Mechanism of TSP Solving Service in Cloud Computing [J]. Computer Science, 2011, 38(12): 194-199.

[4] Rao Weizhen, Jin Chun, Huang Yingyi. Hybrid algorithm of the nearest neighbor and insert ion for the traveling salesman problem [J]. Systems Engineering-Theory \& Practice, 2011, 31(8): 1419-1428. 